\title{
Depressive symptoms and C-reactive protein in a Brazilian urban community
}

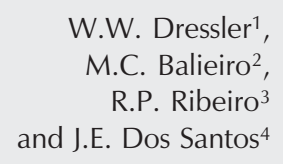

W.W. Dressler ${ }^{1}$,

M.C. Balieiro ${ }^{2}$,

R.P. Ribeiro ${ }^{3}$

and J.E. Dos Santos ${ }^{4}$

\author{
${ }^{1}$ Department of Anthropology, The University of Alabama, Tuscaloosa, AL, USA \\ ${ }^{2}$ Departamento de Psicologia, Universidade Paulista, Ribeirão Preto, SP, Brasil \\ ${ }^{3}$ Escola de Enfermagem de Ribeirão Preto, Universidade de São Paulo, Ribeirão Preto, \\ SP, Brasil \\ ${ }^{4}$ Departamento de Clínica Médica, Faculdade de Medicina de Ribeirão Preto, \\ Universidade de São Paulo, Ribeirão Preto, SP, Brasil
}

\section{Correspondence \\ W.W. Dressler \\ The University of Alabama \\ P.O. Box 870210 \\ Tuscaloosa, AL 35487-0210 \\ USA \\ Fax: +1-205-348-7939 \\ E-mail: wdressle@as.ua.edu \\ Research supported by the National Science Foundation (grant BCS-0090193).}

Publication supported by FAPESP. .........................

Received July 29, 2005 Accepted June 8, 2006

\begin{abstract}
Psychological depression is an independent risk factor for coronary artery disease. C-reactive protein has been implicated as a mediator of the effect of psychological depression. Several studies have found that individuals, especially men, who report higher levels of psychological depression also have higher levels of C-reactive protein. The current study was undertaken to replicate these results in a Brazilian population, in which there is a much wider range of variation in both background characteristics (such as socioeconomic status) and coronary artery disease risk factors. A sample of 271 individuals was interviewed using the Center for Epidemiological Studies Depression Scale. Fasting blood samples were obtained and evaluated for Creactive protein (assessed by a turbidimetric immunoassay using a Dade Behring kit) analysis in a subsample $(\mathrm{N}=258)$ of individuals. The mean \pm SD C-reactive protein for the entire sample was $0.43 \pm$ 0.44 , with $0.42 \pm 0.48$ for men and $0.43 \pm 0.42 \mathrm{mg} / \mathrm{L}$ for women. Data were analyzed using multiple regression analysis, controlling for age, sex, body mass index, socioeconomic status, tobacco use, and both total cholesterol and low-density lipoprotein cholesterol. Higher reported depressive symptoms were correlated with higher $\mathrm{C}$-reactive protein for men (partial $\mathrm{r}=0.298, \mathrm{P}=0.004$ ) and with lower $\mathrm{C}$-reactive protein for women (partial $\mathrm{r}=-0.154, \mathrm{P}=0.059$ ). The differences in the associations for men and women could be a result of differential effects of sex hormones on stress reactivity and immune response. On the other hand, this difference in the associations may be related to gender differences in the disclosure of emotion and the effect that selfdisclosure has on physical health and immune response.
\end{abstract}

Results from several large-scale prospective studies have shown that psychological depression, either in terms of the clinical diagnosis of depression or elevated depressive symptoms on a screening tool, is a risk factor for acute coronary artery disease, and
Key words

- C-reactive protein

- Depressive symptoms

- Brazil

- Gender differences

..................... this risk is independent of standard risk factors (e.g., total cholesterol (TC), systolic blood pressure, smoking). For example, using the Center for Epidemiological Studies Depression Scale (CES-D), Rowan et al. (1) found that a one standard deviation increase 
in depressive symptoms was associated with a $32 \%$ increase in the risk of a coronary event in the Nova Scotia Health Survey. In an Italian study, Marzari et al. (2) observed a doubling of risk associated with depressive symptoms. Meta-analyses of prospective population studies of coronary artery disease have found an aggregated relative risk of 1.64 between high depressive symptoms and subsequent development of coronary artery disease (3).

A number of studies have attempted to elucidate the mechanism underlying this risk, and one promising finding is the association between C-reactive protein (CRP) and depression. Elevated levels of CRP (e.g., >10.0 $\mathrm{mg} / \mathrm{L}$ ) are present during phases of acute inflammation, but studies of coronary artery disease have focused instead on chronic, low-level inflammation. This chronic inflammation is associated with coronary events in persons with unstable angina and with lower survival rates in these patients (4). This has led to the hypothesis that chronic, low-grade inflammation can be implicated in the process of atherosclerosis. It has been suggested that this chronic inflammation contributes to plaque formation and the deposit of plaque on the artery wall. Several studies have found that higher levels of depressive symptoms are associated with higher levels of CRP, especially in men (5-7). CRP thus may represent the physiologic link in this psychobiological process.

The aim of the present study was to determine if the association between depressive symptoms and CRP can be detected in a Brazilian population. Such a study is important for a number of reasons, not the least of which is that within the Brazilian society there is much greater variability in a number of factors (including variability in socioeconomic status and the consumption of dietary fat) that might influence the risk of coronary artery disease. Given this greater range of variability, is it possible to still detect the association of depressive symptoms and CRP?
Data were collected in a community study of cardiovascular disease risk factors conducted in Ribeirão Preto, Brazil, a city of approximately 500,000 inhabitants in the State of São Paulo. To insure that a suitable range of variability in the city was studied, we sampled four neighborhoods that can be ranked along a continuum of socioeconomic status. The poorest neighborhood (which can be referred to as the lower class neighborhood) originally had been a favela (or squatter settlement), but was transformed when the population of the neighborhood was moved by the municipality to a housing project. The second neighborhood was a classic conjunto habitacional (or state-sponsored planned community) that was developed in the late 1980's, and has grown since into a large and stable lower middle-class neighborhood. The third neighborhood was a traditionally middle-class area in the city and the fourth neighborhood was a recently developed upper-middle class neighborhood attractive to professionals and business persons. These neighborhoods have been described in greater detail elsewhere (8).

Complete enumeration of occupied households based on municipal services was obtained for each neighborhood, and simple random samples of households were selected (40 in each neighborhood). Residents first received letters describing the research, and then a visit from a research assistant to describe the research in more detail and to obtain written informed consent. The research protocol was approved by the Institutional Review Board for the Protection of Human Subjects of The University of Alabama, and by the Ethics Committee of the Faculty of Medicine, University of São Paulo, Ribeirão Preto, SP, Brazil. Both the head of household and spouse, and one child over the age of 18 years, were invited to participate in the research. If all members of a household refused participation, a new household was substituted at random. There were 271 persons who participated in the study, 
representing a total response rate of $72.3 \%$. No sample weights were used in the research because the primary aim was to maximize the internal validity of the study, and not to estimate population parameters.

Each respondent was interviewed four times. In the first interview, we collected demographic, sociocultural, and psychological data. The second and third interviews consisted of 24-h dietary recall interviews. One of these interviews was always conducted on a Monday, in order to capture eating patterns over the weekend. The other was conducted on other days of the week. The fourth interview was used to collect clinical data, including anthropometric data, arterial blood pressure, and a fasting blood sample. The sample size for this analysis is limited by the number of people for whom CRP was analyzed $(\mathrm{N}=259)$.

CRP was measured by a turbidimetric immunoassay using a Dade Behring (Deerfield, IL, USA) kit (Flex reagent cartridge Ref. DF34). All determinations were carried out in two assay batches using the same kit. For the assays, standards provided by the manufacturer were used and calibration for each assay utilized doses of 0.0, 2.00, 4.00, 12.00 , and $26.00 \mathrm{mg} / \mathrm{dL}$ CRP. The coefficient of variation within assays was $2 \%$, and the sensitivity of the method was $5 \%$.

Age (in years), body mass index (BMI; $\mathrm{kg} / \mathrm{m}^{2}$ ), and a summary measure of socioeconomic status (SES) were used as control variables. Heights and weights used to calculate BMI were collected with a clinical scale accurate to within $\pm 100 \mathrm{~g}$ and \pm 0.5 $\mathrm{cm}$. SES is a composite variable of family income (in minimum salaries per month), highest level of schooling achieved by the respondent (in years), and occupational status (coded according to the method of Pastore) (9). The composite was calculated as the first principal component score of these variables. Tobacco use was coded as a dichotomy (not currently using $=0$; currently using $=1$ ).

Sex was originally coded as female $=0$ and male $=1$. Depressive symptoms were assessed using the CES-D, translated into Brazilian Portuguese and validated by da Silveira and Jorge (10). This is a 20-item scale of common symptoms of depression, and respondents are asked to indicate the frequency of those symptoms over the preceding two weeks.

Additionally, TC was obtained by an enzymatic procedure (Roche Diagnostic GmbH, Mannheim, Germany) using a Cobas Mira S autoanalyzer (Roche), and low-density lipoprotein cholesterol (LDLc) levels were estimated by the method of Friedewald et al. (11).

Table 1 shows descriptive statistics for all variables included in the analysis for the sample as a whole, and broken down by sex and neighborhood. The large difference in SES indicates that the sampling strategy to enhance variability in the sample was successful. Also, inspecting the descriptive statistics revealed one case, a woman, with a CRP level of $12.0 \mathrm{mg} / \mathrm{L}$. This is probably indicative of an active localized infection, and this case was dropped from the analysis, leaving a total of 258 cases for analysis.

To examine the association of depressive symptoms and CRP, hierarchical multiple regression analysis was used. Also, because previous research has found a difference in the association for males and females, a cross-product term to test for the interaction of sex and depressive symptoms was included, using the method of Cohen and Cohen (12). Sex was converted to contrast codes (females $=-1$ and males $=+1$ ), depressive symptoms were converted to z-scores, and a cross-product term was formed by multiplying sex $\mathrm{x}$ depressive symptoms. Variables were entered into the analysis in sets in the following order: a) age, BMI, tobacco use, and SES; b) sex, depressive symptoms, and the cross-product term, and c) TC or LDLc (in separate analyses). The following results were obtained (reporting standardized or $\beta$ weights): age ( $\beta=0.020$, n.s.); BMI ( $\beta=$ 
$0.315, \mathrm{P}<0.001)$; SES $(B=-0.168, \mathrm{P}<$ $0.01)$; tobacco use $(B=0.013$, n.s.); sex $(\beta=$ -0.020 , n.s.); depressive symptoms $(B=$ 0.108 , n.s.); sex x depressive symptoms ( $\beta=$ $0.236, \mathrm{P}<0.001)$, and, when entered, TC $(B$ $=0.091$, n.s. $)$ or when entered, LDLc $(B=$ $0.127, \mathrm{P}=0.055)$. For each model, the multiple $\mathrm{R}$ was $0.455(\mathrm{P}<0.001)$.

Additional analyses were carried out. Substituting abdominal circumference for BMI, or using log-transformed values of CRP, made no substantive difference in the results. Influential case analyses (12) indi- cated three influential cases (two with large leverage values and one with a large studentized deleted residual). But the results remained substantially the same when these cases were deleted.

The pattern of the statistical interaction effect between sex and depressive symptoms is shown in Figure 1 (CRP was adjusted for age, BMI, SES, and LDLc using linear regression). For men, there was a statistically significant correlation between higher depressive symptoms and higher CRP $(\mathrm{P}=0.004)$; as depressive symptoms in-

Table 1. Descriptive statistics of the population studied.

\begin{tabular}{|c|c|c|c|c|c|}
\hline & $\begin{array}{l}\text { Lower class } \\
\text { neighborhood } \\
(\mathrm{N}=58)\end{array}$ & $\begin{array}{l}\text { Working class } \\
\text { neighborhood } \\
(\mathrm{N}=64)\end{array}$ & $\begin{array}{c}\text { Middle-class } \\
\text { neighborhood } \\
(\mathrm{N}=69)\end{array}$ & $\begin{array}{l}\text { Upper-middle class } \\
\text { neighborhood } \\
\qquad(\mathrm{N}=67)\end{array}$ & $\begin{array}{c}\text { Total } \\
(\mathrm{N}=258)\end{array}$ \\
\hline C-reactive protein $(\mathrm{mg} / \mathrm{L})^{*}$ & $0.38 \pm 0.34$ & $0.55 \pm 0.49$ & $0.48 \pm 0.58$ & $0.31 \pm 0.26$ & $0.43 \pm 0.44$ \\
\hline Men & $0.43 \pm 0.39$ & $0.56 \pm 0.53$ & $0.45 \pm 0.63$ & $0.27 \pm 0.22$ & $0.42 \pm 0.48$ \\
\hline Women & $0.36 \pm 0.31$ & $0.54 \pm 0.47$ & $0.50 \pm 0.54$ & $0.33 \pm 0.26$ & $0.43 \pm 0.42$ \\
\hline Age $\left(\right.$ years) ${ }^{*}$ & $36.8 \pm 10.3$ & $43.5 \pm 11.5$ & $41.8 \pm 11.9$ & $41.3 \pm 12.1$ & $40.9 \pm 11.7$ \\
\hline Men & $37.4 \pm 10.5$ & $43.8 \pm 11.8$ & $40.0 \pm 11.4$ & $41.7 \pm 12.7$ & $41.0 \pm 11.7$ \\
\hline Women & $36.6 \pm 10.3$ & $42.9 \pm 11.4$ & $43.1 \pm 12.3$ & $41.0 \pm 11.8$ & $40.8 \pm 11.7$ \\
\hline Sex (\% men) & $29.3 \%$ & $37.5 \%$ & $44.9 \%$ & $41.8 \%$ & $38.8 \%$ \\
\hline Body mass index $\left(\mathrm{kg} / \mathrm{m}^{2}\right)^{*}$ & $24.4 \pm 5.0$ & $26.4 \pm 5.7$ & $25.7 \pm 4.9$ & $24.1 \pm 5.0$ & $25.2 \pm 5.2$ \\
\hline Men & $24.5 \pm 3.1$ & $26.7 \pm 4.8$ & $25.3 \pm 5.2$ & $26.0 \pm 5.7$ & $25.7 \pm 5.0$ \\
\hline Women & $24.4 \pm 5.7$ & $26.2 \pm 6.4$ & $26.0 \pm 4.7$ & $22.8 \pm 3.9$ & $24.8 \pm 5.4$ \\
\hline Socioeconomic status (standardized factor score) ${ }^{*}$ & $-0.85 \pm 0.65$ & $-0.21 \pm 0.64$ & $-0.09 \pm 0.88$ & $1.14 \pm 0.47$ & $0.02 \pm 0.98$ \\
\hline Men & $-0.90 \pm 0.57$ & $-0.20 \pm 0.69$ & $0.02 \pm 0.86$ & $1.19 \pm 0.50$ & $0.13 \pm 0.99$ \\
\hline Women & $-0.84 \pm 0.68$ & $-0.22 \pm 0.61$ & $-0.19 \pm 0.89$ & $1.10 \pm 0.45$ & $-0.05 \pm 0.97$ \\
\hline Depressive symptoms (symptom frequency)* & $14.9 \pm 8.9$ & $11.5 \pm 9.2$ & $13.7 \pm 12.1$ & $9.2 \pm 8.3$ & $12.2 \pm 10.0$ \\
\hline Men & $9.8 \pm 6.4$ & $10.9 \pm 9.7$ & $12.1 \pm 10.8$ & $7.8 \pm 6.7$ & $10.2 \pm 8.9$ \\
\hline Women** & $17.0 \pm 9.0$ & $11.8 \pm 9.0$ & $15.0 \pm 13.1$ & $10.3 \pm 9.2$ & $13.5 \pm 10.4$ \\
\hline Tobacco use $(\% \text { using })^{+}$ & $34.5 \%$ & $15.6 \%$ & $26.1 \%$ & $10.4 \%$ & $21.3 \%$ \\
\hline Men & $41.2 \%$ & $16.7 \%$ & $29.0 \%$ & $7.1 \%$ & $22.0 \%$ \\
\hline Women & $31.7 \%$ & $15.0 \%$ & $23.7 \%$ & $12.8 \%$ & $20.9 \%$ \\
\hline Total cholesterol $(\mathrm{mg} / \mathrm{dL})^{*}$ & $181.4 \pm 36.2$ & $185.8 \pm 40.9$ & $176.9 \pm 33.5$ & $204.4 \pm 36.2$ & $187.2 \pm 38.0$ \\
\hline Men & $190.2 \pm 47.1$ & $197.2 \pm 41.5$ & $173.2 \pm 37.1$ & $209.5 \pm 36.3$ & $192.0 \pm 41.7$ \\
\hline Women & $177.7 \pm 30.5$ & $178.9 \pm 39.5$ & $179.9 \pm 30.4$ & $200.7 \pm 36.1$ & $184.2 \pm 35.3$ \\
\hline Low-density lipoprotein cholesterol (mg/dL)* & $105.9 \pm 29.6$ & $111.0 \pm 34.3$ & $100.0 \pm 29.2$ & $132.3 \pm 32.5$ & $112.6 \pm 33.7$ \\
\hline Men & $111.9 \pm 31.7$ & $120.4 \pm 37.5$ & $97.6 \pm 29.7$ & $142.4 \pm 32.4$ & $118.5 \pm 36.8$ \\
\hline Women** & $103.5 \pm 28.8$ & $105.4 \pm 31.3$ & $101.8 \pm 29.1$ & $124.9 \pm 31.0$ & $108.8 \pm 31.2$ \\
\hline
\end{tabular}

Data are reported as mean \pm SD or as percent.

*Differences in these parameters between neighborhoods are significant $\left(P<0.05\right.$, ANOVA). ${ }^{*}$ Differences in these parameters between men and women are significant $\left(P<0.05\right.$, ANOVA). ${ }^{+}$Differences in these parameters between neighborhoods are significant $(P<0.05$, chi-square test). 
crease, CRP also increases. For women, this correlation was weakly inverse and achieved borderline statistical significance $(\mathrm{P}=0.059)$.

The results obtained here replicate those obtained in other studies in other societies. First, there was an association between depressive symptoms and CRP. Second, this association was positive for men, and weakly inverse for women. Third, this association was statistically independent of other factors, especially BMI, SES, and LDLc, that might affect the association. It can be suggested, therefore, that in this Brazilian population, depressive symptoms may increase the risk of coronary artery disease for men by increasing the likelihood of a chronic, low-grade, systemic inflammation.

The psychophysiologic pathway by which depressive symptoms influence CRP is probably through the hypothalamic-pituitary-adrenal (HPA) axis. Many, but not all, forms of depression are accompanied by an increase in circulating levels of corticosteroids, and, through a heightened sensitivity to stressful events, by elevated catecholamine levels, both of which can stimulate release of cytokines, especially the pro-inflammatory interleukin 6. Interleukin 6 in turn is an important inducer of CRP by the liver. Along with stress-induced changes in the artery walls mediated by elevated blood pressure, this process can promote the deposit of plaques on the artery wall (13). It is important to emphasize, however, that the effects of stressors and mood states are neither universally suppressive nor stimulating with respect to the immune system. A better concept is one of "immune dysregulation", in which stressors and affective responses to those stressors can lead to complex interactions among the HPA axis, the central nervous system, and affected target organs and systems (14).

What complicates this argument is that the association of depressive symptoms and CRP is different for men and women. There may be an interaction of sex hormones with the HPA axis that affects this process (15).
At the same time, there may be sociocultural factors intervening as well. A widely replicated observation in the study of depressive symptoms is the difference in symptom number and intensity expressed by women versus men, and a higher prevalence of clinical depression among women than among men (16). This difference has been attributed to differences in the cultural appropriateness of expressions of affect-negative or positivebetween men and women. In many societies, the expression of emotion by women is considered to be socially and culturally more appropriate than is the expression of emotion by men (17). This has been used to explain sex differences in depression; women may more readily admit to feelings, including the negative feelings of depression, than men. In Brazil, women report higher levels of psychological distress than men (18), and there is some evidence of a particular intensity of the reported experience of emotion in Brazilian samples (19).

This fact could help to explain the differ-

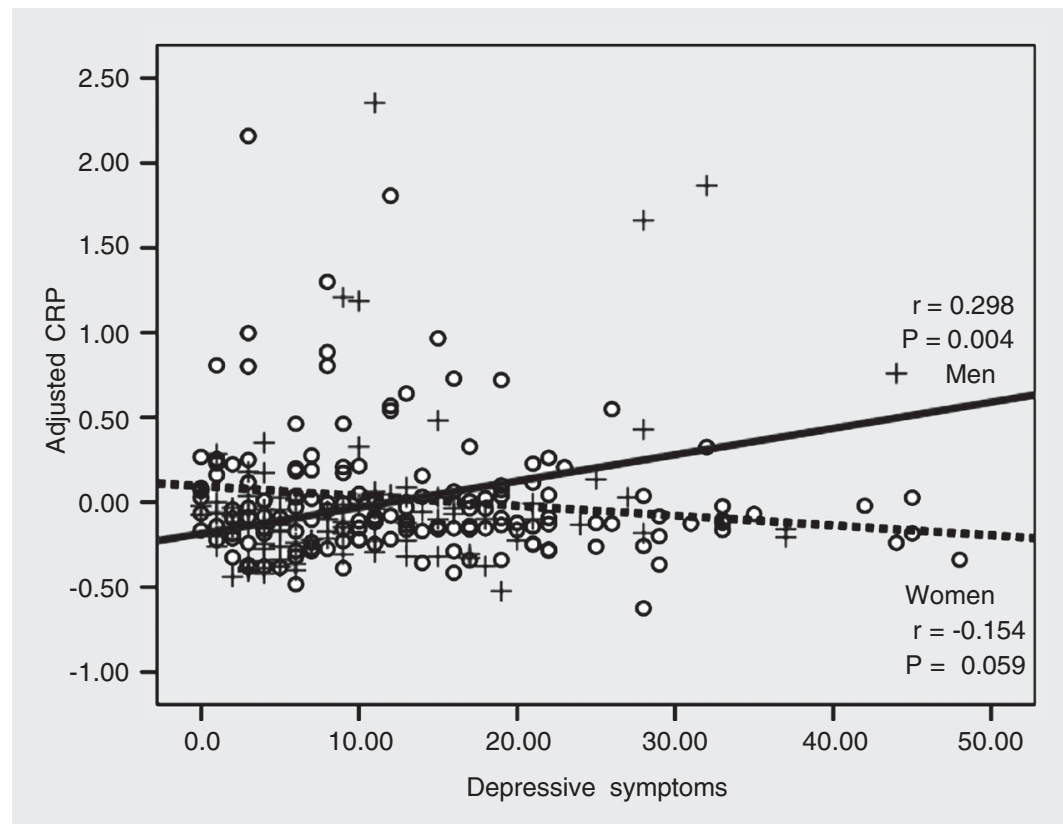

Figure 1. Association of depressive symptoms and C-reactive protein (CRP), adjusted for age, body mass index, socioeconomic status, and low-density lipoprotein cholesterol (adjusted by linear regression). Plus signs and solid line = men $(N=100)$; circles and dashed line $=$ women $(N=158)$. 
ence in the association of depressive symptoms and CRP between men and women. A number of studies have demonstrated the beneficial effects of self-disclosure during times of stress. That is, persons who, during times of stress, express their distress consciously and verbally also suffer fewer repercussions of that stress, both psychologically and physiologically. Pennebaker (20) in particular has shown that persons who disclose the impact and meaning of stressful events suffer fewer negative physical consequences. Perhaps for women, the cultural acceptance of their expressions of emotion in depression helps to blunt the impact of that emotion through the HPA axis, resulting in turn in better functioning of the immune system and less chronic inflammation. For men, on the other hand, the culturally sanctioned suppression of emotion may result in increased stress, chronic systemic inflammation, and a greater risk of atherogenesis. Note, too, that in our data, men are not spontaneously reporting the experience of depressive symptoms, but rather are responding to a series of questions in a research setting. It is likely that many of our male respondents would not spontaneously discuss their feelings.

This of course is speculative, but it is consistent with what is known about differences in the expression of emotion between men and women, and the positive effects of self-disclosure. It should be noted, however, that the cross-sectional nature of our findings does not allow us to distinguish between the effects of depression on CRP and the effects of CRP on depression, although discussions in the literature consistently emphasize the direction of causation as depression to CRP.

There are a number of methodological issues that could affect the findings. First, different interviewers might be more effective in eliciting reports of depressive symptoms from respondents, and if these respondents in turn differed in CRP, the results could be spurious; however, there were no differences in symptom reports between the two interviewers in the study, nor did they interview different proportions of men and women. Second, as noted above, we do not have a simple random sample of the population, but rather a stratified random sample designed to insure the inclusion of higher income respondents. This could possibly affect the estimate of the relationship between CRP and depressive symptoms in the population; however, our aim here was not to estimate population parameters, but rather to employ a sample that would be more effective in taking into account the wellknown socioeconomic variability in Brazilian communities and hence provide a stronger test of the hypothesis by insuring the representation of upper-income groups. We did, however, replicate these analyses using sample weights based on the income distribution in the study community, in order to adjust the sample statistically to resemble more the overall population. There were no differences in the results using these sample weights. Nevertheless, it would be useful to repeat this study in samples designed to be more broadly representative of the overall population.

What these findings help to underscore is the importance of the social environment and cultural factors in the production of cardiovascular disease. As we have shown elsewhere, persons who are less successful in meeting widely shared cultural expectations - a factor we refer to as "cultural consonance" - also are at a much higher risk of psychological depression (8). Our results here and in other papers thus suggest a process in which atherosclerosis and the risk of acute coronary artery disease start "upstream" in the adaptation of the individual to his social and cultural environment. The individual who is less culturally consonant is at higher risk of experiencing more symptoms of depression, and, we hypothesize, if he is inhibited from expressing that emotion be- 
cause of cultural expectation, he is, in turn, at a higher risk of chronic systemic inflammation, ultimately increasing the risk of atherosclerosis and a coronary event. On the other hand, expressing emotion may be associated with a different physiologic representation of that emotion that reduces the risk of chronic inflammation and a subsequent coronary event. This is, in other words, a truly biocultural process. The results pre- sented here help to round out our understanding of the steps in this process, from the societal to the molecular.

\section{Acknowledgments}

\section{Dr. Thomas McDade (Northwestern Uni-} versity) provided helpful comments on an earlier draft of this paper.

\section{References}

1. Rowan PJ, Haas D, Campbell JA, Maclean DR, Davidson KW. Depressive symptoms have an independent, gradient risk for coronary heart disease incidence in a random, population-based sample. Ann Epidemiol 2005; 15: 316-320.

2. Marzari C, Maggi S, Manzato E, Destro C, Noale M, Bianchi D, et al. Depressive symptoms and development of coronary heart disease events: the Italian longitudinal study on aging. J Gerontol A Biol Sci Med Sci 2005; 60: 85-92.

3. Rugulies R. Depression as a predictor for coronary heart disease. A review and meta-analysis. Am J Prev Med 2002; 23: 51-61.

4. Pai JK, Pischon T, Ma J, Manson JE, Hankinson SE, Joshipura K, et al. Inflammatory markers and the risk of coronary heart disease in men and women. N Engl J Med 2004; 351: 2599-2610.

5. Danner M, Kasl SV, Abramson JL, Vaccarino V. Association between depression and elevated C-reactive protein. Psychosom Med 2003; 65: 347-356.

6. Ford DE, Erlinger TP. Depression and C-reactive protein in US adults: data from the Third National Health and Nutrition Examination Survey. Arch Intern Med 2004; 164: 1010-1014.

7. Suarez EC. C-reactive protein is associated with psychological risk factors of cardiovascular disease in apparently healthy adults. Psychosom Med 2004; 66: 684-691.

8. Dressler WW, Ribeiro RP, Balieiro MC, Oths KS, Dos Santos JE. Eating, drinking and being depressed: the social, cultural and psychological context of alcohol consumption and nutrition in a Brazilian community. Soc Sci Med 2004; 59: 709-720.

9. Pastore J. Inequality and social mobility in Brazil. Madison: University of Wisconsin Press; 1982.

10. da Silveira DX, Jorge MR. Escala de rastreamento populacional para depressão (CES-D) em populações clínica e não clínica de adolescentes e adultos jovens. In: Gorenstein C, Andrade LHSG, Zuardi AW (Editors), Escalas de avaliação clínica em psiquiatria e psicofarmacologia. São Paulo: Lemos-Editorial; 2000.

11. Friedewald WT, Levy RI, Fredrickson DS. Estimation of the concentration of low-density lipoprotein cholesterol in plasma, without use of the preparative ultracentrifuge. Clin Chem 1972; 18: 499-502.

12. Cohen J, Cohen P. Applied multiple regression/correlation analysis for the behavioral sciences. 2nd edn. Hillsdale: Lawrence Erlbaum Associates; 1983.

13. Black $\mathrm{PH}$. The inflammatory response is an integral part of the stress response: Implications for atherosclerosis, insulin resistance, type II diabetes and metabolic syndrome X. Brain Behav Immun 2003; 17: 350-364.

14. Glaser R, Kiecolt-Glaser JK. Stress-induced immune dysfunction: implications for health. Nat Rev Immunol 2005; 5: 243-251.

15. Young EA. Sex differences and the HPA axis: implications for psychiatric disease. J Gend Specif Med 1998; 1: 21-27.

16. Barsky AJ, Peekna HM, Borus JF. Somatic symptom reporting in women and men. J Gen Intern Med 2001; 16: 266-275.

17. Simon RW, Nath LE. Do men and women differ in self-reports of feelings and expressive behavior? Am J Sociol 2004; 109: 11371176.

18. de Almeida-Filho N. Migration and mental health in Bahia, Brazil. Zaragoza: Caja de Ahorros de la Inmaculada; 1987.

19. Ribeiro RL, Pompeia S, Bueno OF. Comparison of Brazilian and American norms for the International Affective Picture System (IAPS). Rev Bras Psiquiatr 2005; 27: 208-215.

20. Pennebaker JW. Putting stress into words: health, linguistic, and therapeutic implications. Behav Res Ther 1993; 31: 539-548. 